ARTICLE

\title{
Systems genetics identifies a role for Cacna2d1 regulation in elevated intraocular pressure and glaucoma susceptibility
}

Sumana R. Chintalapudi ${ }^{1}$, Doaa Maria ${ }^{1,2,3}$, Xiang Di Wang ${ }^{1}$, Jessica N. Cooke Bailey (D) ${ }^{4}$, NEIGHBORHOOD consortium, International Glaucoma Genetics consortium, Pirro G. Hysi ${ }^{5}$, Janey L. Wiggs ${ }^{6}$, Robert W. Williams ${ }^{1,7,8} \&$ Monica M. Jablonski (i) ${ }^{1,2,7}$

Glaucoma is a multi-factorial blinding disease in which genetic factors play an important role. Elevated intraocular pressure is a highly heritable risk factor for primary open angle glaucoma and currently the only target for glaucoma therapy. Our study helps to better understand underlying genetic and molecular mechanisms that regulate intraocular pressure, and identifies a new candidate gene, Cacna2d1, that modulates intraocular pressure and a promising therapeutic, pregabalin, which binds to CACNA2D1 protein and lowers intraocular pressure significantly. Because our study utilizes a genetically diverse population of mice with known sequence variants, we are able to determine that the intraocular pressure-lowering effect of pregabalin is dependent on the Cacna2d7 haplotype. Using human genome-wide association study (GWAS) data, evidence for association of a CACNA2D1 single-nucleotide polymorphism and primary open angle glaucoma is found. Importantly, these results demonstrate that our systems genetics approach represents an efficient method to identify genetic variation that can guide the selection of therapeutic targets.

\footnotetext{
${ }^{1}$ Department of Ophthalmology, The Hamilton Eye Institute, The University of Tennessee Health Science Center, Memphis, TN 38163, USA. ${ }^{2}$ Department of Pharmaceutical Sciences, The University of Tennessee Health Science Center, Memphis, TN 38163, USA. ${ }^{3}$ Department of Pharmaceutics, College of Pharmacy, Mansoura University, Mansoura 35516, Egypt. ${ }^{4}$ Department of Epidemiology and Biostatistics, Institute of Computational Biology, Case Western Reserve University School of Medicine, Cleveland, OH 44106, USA. ${ }^{5}$ Department of Twin Research and Genetic Epidemiology, King's College London, London WC2R 2LS, UK. ${ }^{6}$ Department of Ophthalmology, Harvard Medical School, Massachusetts Eye and Ear Infirmary, Boston, MA 02298, USA. ${ }^{7}$ Department of Anatomy and Neurobiology, The University of Tennessee Health Science Center, Memphis, TN 38163, USA. ${ }^{8}$ Department of Genetics, Genomics and Informatics, The University of Tennessee Health Science Center, Memphis, TN 38163, USA. Correspondence and requests for materials should be addressed to M.M.J. (email: mjablonski@uthsc.edu)

A full list of authors and their affliations appears at the end of the paper.
} 
rimary open angle glaucoma (POAG) is a leading cause of blindness worldwide ${ }^{1}$. The disease is characterized by progressive optic nerve damage arising from apoptotic cell death of retinal ganglion cells. Elevated intraocular pressure (IOP) is one of the most significant risk factors contributing to visual field loss in this disease. Steady-state IOP is generated by the balance of aqueous humor $(\mathrm{AH})$ production by the ciliary body (CB) and $\mathrm{AH}$ drainage through the trabecular meshwork (TM; conventional pathway), and to a lesser degree the uveoscleral or nonconventional pathway. An imbalance between the inflow and outflow of $\mathrm{AH}$ leads to a change in $\mathrm{IOP}^{2-4}$. Gene variants influence an individual's likelihood of developing glaucoma, the rate of disease progression, and how a patient responds to treatment. Because IOP can be medically controlled, IOP reduction is the first-line therapeutic option in glaucoma. Current medications do not address the underlying pathologies that lead to elevated IOP, nor do they address the many potential sources of variation related to IOP modulation.

Both POAG and IOP are highly heritable. In humans, IOP heritability is estimated to be $\sim 55 \%$. Moreover, the genetic risk of elevated IOP and POAG are partially shared ${ }^{5,6}$, although some loci that are associated with POAG were not associated with $\mathrm{IOP}^{7}$. To date, multiple candidate IOP or POAG loci have been identified (e.g., TMCO1, CDKN2B-AS1, GAS7, CAV1/CAV2, SIX1/SIX6, TXNRD2, ATXN2, FOXC1 ABCA1, AFAP1, GMDS, PMM2, TGFBR3 ARHGEF12, FAM125B, FNDC3B, and the ABO blood group), however, their physiological roles are not well understood $^{7-16}$. Identification of additional gene variants that modulate IOP both in animals and humans is therefore likely to provide critical insights and new targets for therapeutic intervention.

Systems genetics is an extension of complex trait analysis that examines large sets of genotypes and phenotypes to investigate the genetic basis of disease traits ${ }^{17-20}$. The BXD family is currently the largest and best characterized mouse genetic reference population $^{21}$. This family is an admixture of C57BL/6J and DBA/ 2J genomes, and the family are segregating for roughly 5.5 million sequence variants ${ }^{22}$. Over the last decade, the BXD family has become a key resource for systems genetics largely because they have been phenotyped so thoroughly and at many levels-from messenger RNA (mRNA) to maternal behavior ${ }^{17,23-25}$. There is also extensive phenome data on the visual system of these strains ${ }^{26-30}$. One of the major advantages of the BXDs relative to the Collaborative Cross and other new resources is that the DBA/ 2J progenitor strain and many of the BXD progeny strains consistently develop high IOP between 6 and 10 months of age $\mathrm{e}^{31}$. Therefore, they are an ideal resource to discover gene variants that modulate both IOP and glaucoma.

In this study, we systematically measure IOP across a large subset of the BXD family in multiple age cohorts. Using stringent stepwise refinement based on expression quantitative trait locus (QTL) mapping, correlation analyses (direct and partial Pearson test), and the analysis of single-nucleotide polymorphisms (SNPs), we identify a candidate gene that modulates IOP, and we combine mouse and human genetic data in an effort to validate the candidate gene. Lastly, we evaluate the IOP-lowering effect of a drug specifically targeted to the candidate protein product. Collectively, our study finds that Cacna2d1 modulates IOP and that blocking the function of its gene product, CACNA2D1, with pregabalin reduces IOP in a dose-dependent and haplotypespecific manner.

\section{Results}

Study design. A major advantage of the genetically diverse BXD family over non-inbred strains is that each strain is homozygous

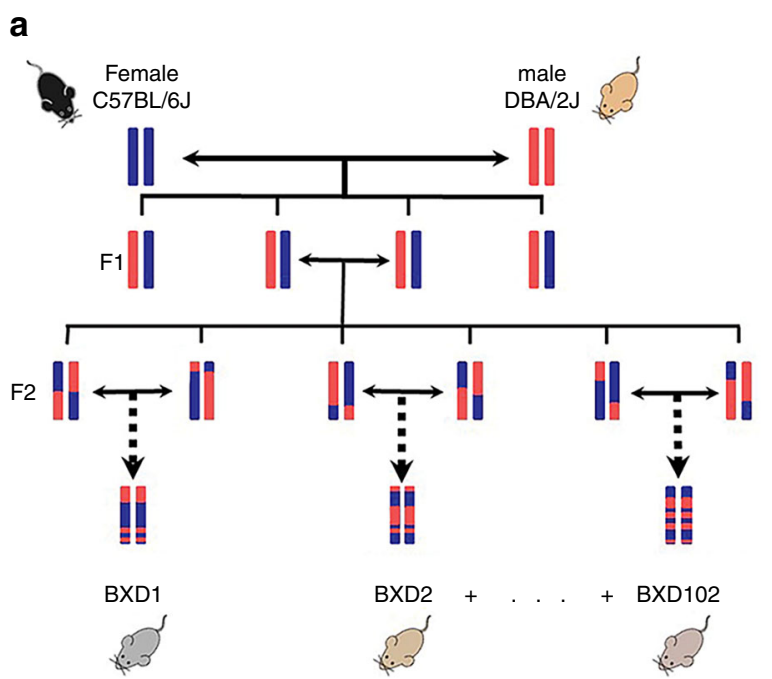

b

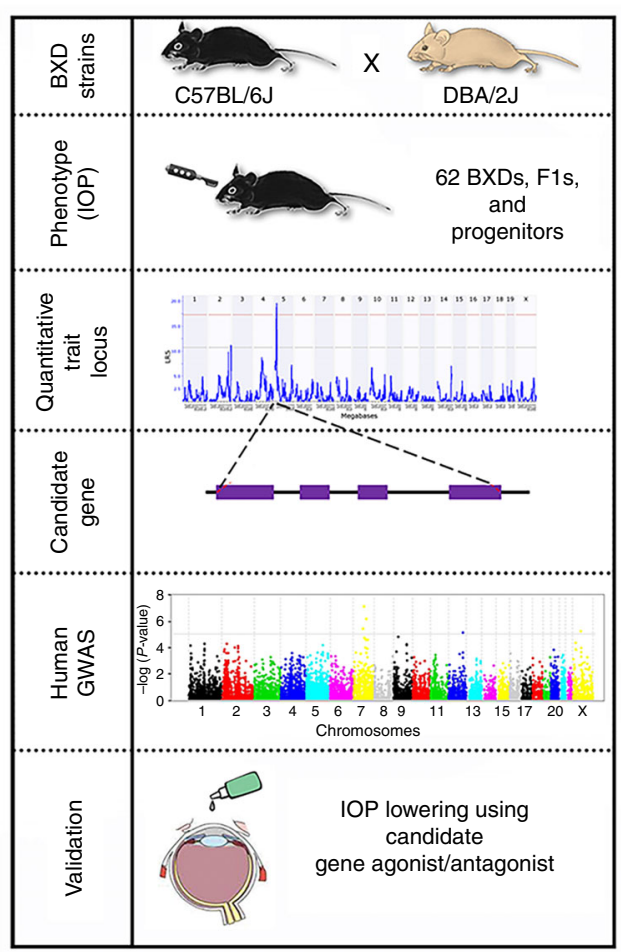

Fig. 1 Overview of IOP systems genetics analyses. a BXD strains derived by crossing C57BL/6J (B6) and DBA/2J (D2) parents. The resulting heterozygous $\mathrm{F} 1$ mice were crossed to generate genetically diverse but nonreproducible F2 animals. These F2 progeny were iteratively inbred until generation $\mathrm{F} 2 \mathrm{O}+$, at which point each strain represents a unique mosaic of $B$ and $D$ alleles. $\mathbf{b}$ Workflow for IOP systems genetics analysis. IOP was measured from 65 BXD strains aged 9.1-13 months. Genomic regions modulating IOP were identified using QTL analyses. Stringent refinement based on QTL mapping, correlation analyses, and SNPs was performed to identify positional candidate genes. Subcellular localization of candidates in mouse and human eyes were determined by immunohistochemistry. The NEIGHBORHOOD consortium database was used to identify SNPs within the candidate gene associated with elevated IOP and POAG in humans. The IOP-lowering effect of pregabalin, a drug with high affinity for CACNA2D1 was evaluated as eye drops in D2, BXD48 ( $D$ allele), B6, and BXD14 ( $B$ allele) strains for validation 
across its genome (Fig. 1a), which allows for an infinite source of genetically identical test subjects. IOP is one factor that can influence glaucoma risk. To identify a gene candidate that modulates IOP, we utilized a systems genetics, pharmacological, and translational approach (Fig. 1b). We have successfully used this approach in previous studies to identify gene modulators of various traits in BXD mice ${ }^{26,27,29,30,32,33 . ~}$

Trait and genetic variation across BXD mice. IOP varied over twofold in 9.1-13-month-old BXD strains (range of $9.6 \pm 0.92$ a

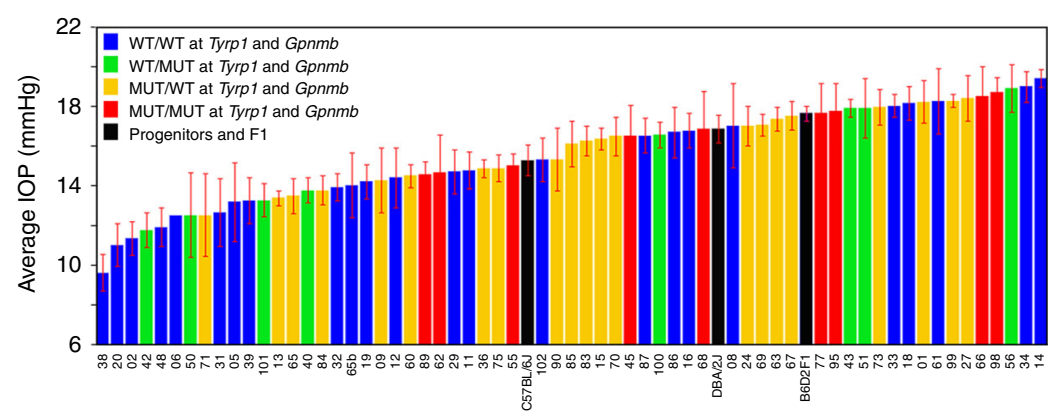

C

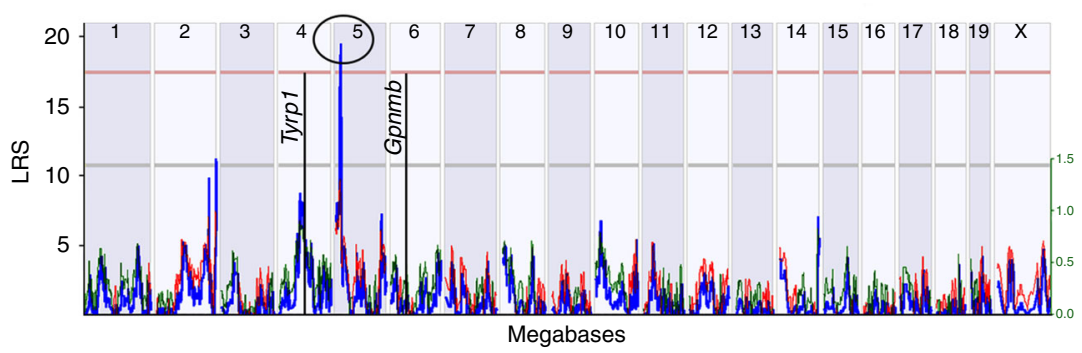

b

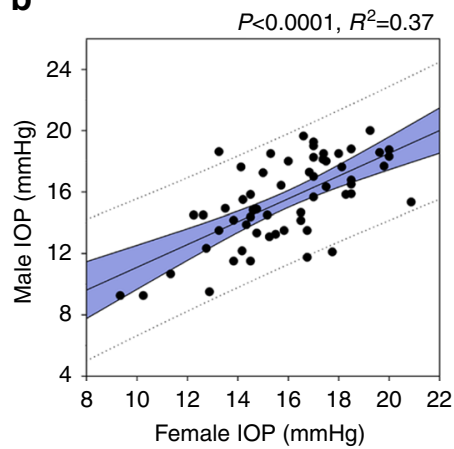

d

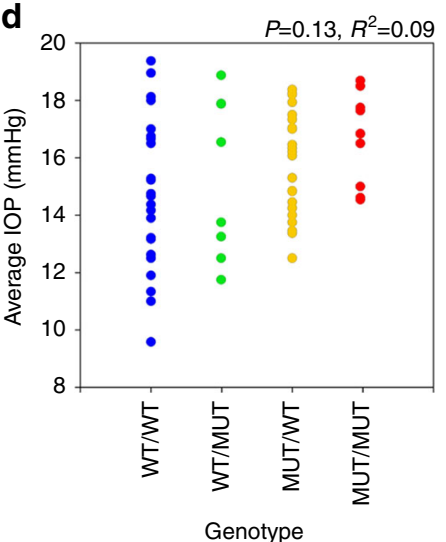

e

Candidate gene selection criteria

1. Location within the confidence interval of the peak eQTL

2. Cis-modulated gene

3. Significant correlation (linear and partial) between expression of the gene and IOP

4. Functions within a network that could explain its role in modulating IOP

5. Presence of sequence variants between parental strains at/near the region of the gene

6. Expression in the eye and localization to a structure associated with modulation of IOP

7. Association with human POAG or IOP through GWAS or standard linkage studies

8. Biological association with glaucoma or its treatment

g

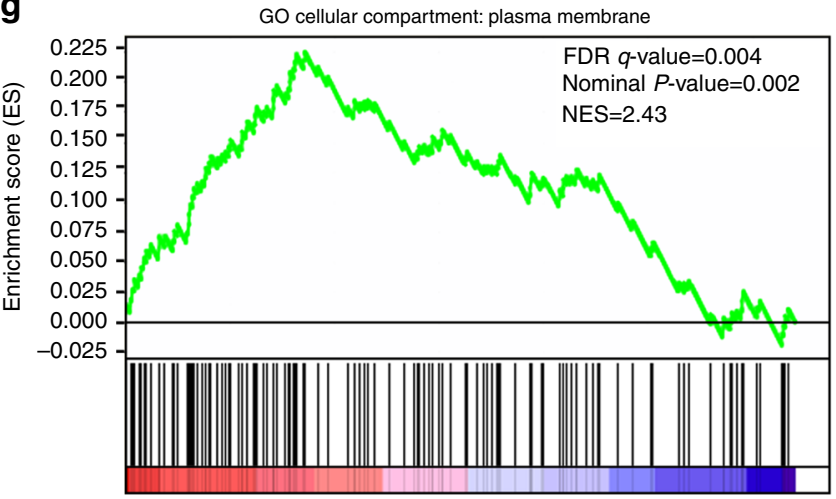

(Pos. correlated with Cacna2d1) (Neg. correlated with Cacna2d1) f

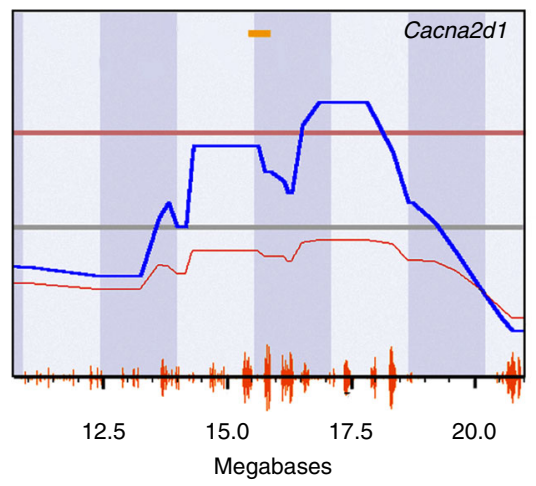

h

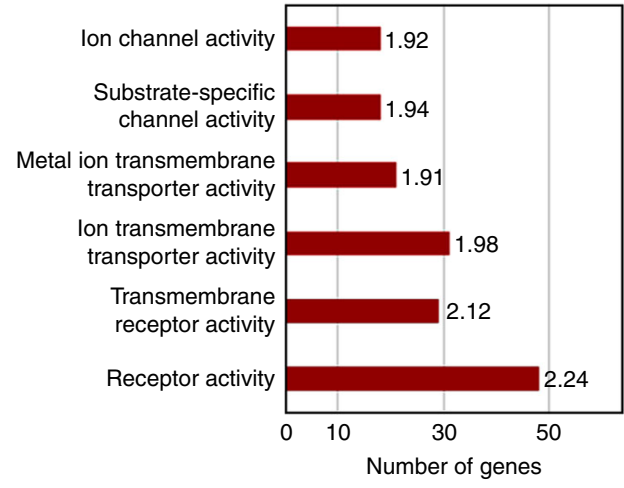


$\mathrm{mm} \mathrm{Hg}$ in BXD38 to $19.38 \pm 0.43 \mathrm{~mm} \mathrm{Hg}$ in BXD14; Fig. 2a). IOP heritability was $31 \%$, which is comparable to that of other ocular traits to which QTL mapping has been applied successfully ${ }^{27}, 29,32-34$. There was no statistically significant difference in IOP between the sexes (Fig. 2b).

The variation in IOP was mapped to a narrow locus on proximal Chr 5 (likelihood ratio statistic (LRS) $=19.6$, logarithm of the odds $(\mathrm{LOD})=4.25$, Fig. 2c). This QTL was not associated with IOP in studies using younger sex-matched BXD strains, suggesting that the QTL contains gene variants that contribute to variation in IOP at an older age ${ }^{29}$. In addition, the location of this LRS interval does not overlap with the location of either Tyrp1 or Gpnmb (Fig. 2c). As confirmation that the well-documented mutations in Tyrp1 and Gpnmb do not influence IOP in the BXD GRP, IOP also maps to the same location on Chr 5 after we exclude strains that harbor both mutations or harbor only one of the mutations (Supplementary Fig. 1). Moreover, the complete overlap of IOP distributions for all possible genotypes at those two loci $(P=0.13$; Fig. 2 d) further supports this claim.

Selection of positional candidates in the chromosome 5 locus. To determine the candidate gene variants that modulate IOP within the Chr 5 locus, we used the following stringent criteria (Fig. 2e): (1) the gene is located within the confidence interval of the peak eQTL; (2) the gene has cis-modulation; (3) the expression level of the gene across BXD strains is significantly correlated with elevated IOP using both linear correlation and partial Pearson correlation analyses; (4) the gene functions within a network that could explain its role in modulating IOP; (5) the gene has sequence variants between parental strains at/near the region of the gene; (6) the gene is expressed in the eye and localized to an area associated with modulation of IOP; (7) the gene is associated with human POAG and/or elevated IOP either through GWAS or standard linkage studies; and (8) the gene has a biological association with glaucoma or its treatment.

Within the QTL peak at Chr 5, there were 25 positional gene candidates that were cis-regulated (Supplementary Table 1). Using our above criteria, calcium channel, voltage-dependent, $\alpha 2 \delta 1$ subunit (Cacna2d1) emerged as the single best positional candidate $(r=0.440 ; P=0.0003$; Fig. $2 \mathrm{f})$. No other positional candidate fulfilled our selection criteria (Supplementary Table 2). Gene set enrichment analyses (GSEA; Broad Institute ${ }^{35}$ ) determined that nominally significant gene correlates of Cacna2d1 (Supplementary Data 1) were significantly enriched for plasma membrane localization (Fig. $2 \mathrm{~g}$ ) and for functions related to neuronal function and excitability, including ion channel activity, substrate-specific channel activity, metal ion transmembrane transporter activity, ion transmembrane transporter activity, and receptor activity (Fig. 2h). These results suggest that
Cacna2d1 likely functions within pathways that critically influence ion channels and/or their receptors within the eye that affect IOP regulation.

Gene haplotypes of Cacna2d1 contribute to variations in IOP. The expression of the Cacna2d1 transcript in the whole eye varied significantly among BXD strains (range of $6.9 \pm 0.2$ in BXD71 to $9.7 \pm 0.3$ in BXD48a), which is a 5.6-fold difference in mRNA expression (Fig. 3a). BXD strains with the $D$ parental allele have lower expression of Cacna2d1 in the eye than those strains with the $B$ parental allele (Fig. 3a, b, $P=1.2 \times 10^{-32}$ ). Cacna2d1 mapped as a significant cis-eQTL (LRS $=143$; LOD $=31.01$ ) on proximal $\mathrm{Chr} 5$ at $14.3 \mathrm{Mb}$, which is within $5 \mathrm{Mb}$ of location of the gene itself (Fig. 3c), making it a cis-regulated gene.

Cacna2d1 encodes for a preproprotein that is cleaved into multiple chains that form the alpha-2 and delta subunits of the voltage-dependent calcium channel complex. CACNA2D1 is a glycosylphosphatidylinositol (GPI)-anchored subunit typically associated with the Cav $\alpha 1$ pore within L-type calcium channels in smooth muscle (Fig. 3d). The gene is highly polymorphic (Fig. 3e) with 1056 SNPs and 30 insertions/deletions between the parent strains (Supplementary Data 2). These variants segregate among the BXD strains based upon the haplotype of the gene. Similarly, in humans, CACNA2D1 is highly polymorphic and has several splice variants ${ }^{36-38}$.

We further sought to determine if the Cacna2d1 haplotype influenced the baseline IOP in BXD mice. Similar to the influence of the parental allele on Cacna2d1 expression (Fig. 3b), we found a distinct segregation of IOP values among the BXD strains that was dependent upon the haplotype of Cacna2d1 that was agedependent. The Cacna2d1 haplotype had no significant effect on IOP in younger BXD strains ( $P=0.34$, age $1-2.1$ months) (Fig. 3f, left). In contrast, in older age (aged 9.1-13 months), strains with Cacna2d1 inherited from the B6 parent ( $B$ allele) had significantly higher IOP than those with the $D$ allele $(P=0.0008$, Fig. 3f, right).

To further assess the strength of this candidate gene, we evaluated human POAG GWAS data from the NEIGHBORHOOD consortium $(N=3853 \text { cases and } 33480 \text { controls })^{16}$. A total of 1520 SNPs in the human genomic region that includes CACNA2D1 (chromosome 7:81.9-82.4 Mb) were evaluated, identifying nominal association for POAG $(P<0.05)$ for 44 SNPs (Supplementary Table 3), with the lead SNP (rs2299184 $[\mathrm{A}], P=0.001$, odds ratio $=1.15)$ located in intron 1 near DNaseI hypersensitivity sites annotated by the Encyclopedia of DNA Elements (ENCODE) as active in non-pigmented ciliary epithelium. Eight of the 44 human CACNA2D1 SNPs showing nominal association in the NEIGHBORHOOD POAG GWAS were also included in a multi-ethnic IOP association study ${ }^{9}$, however,

\footnotetext{
Fig. 2 Association analyses reveal Cacna2d1 as a candidate for IOP modulation. a IOP levels vary among the BXD strains. IOP of BXD strains carrying wild-type alleles of Tyrp1 and Gpnmb (WT/WT; blue bars), wild-type Tyrp1 and mutant Gpnmb (WT/MUT; green bars), mutant Tyrp1 and WT Gpnmb (MUT/WT; yellow bars), and mutant alleles of both genes (MUT/MUT; red bars) are shown. $n=65$ strains, age $=9.1-13$ months. Values denote IOP levels on $\mathrm{mmHg}$ scale (mean $\pm \mathrm{SEM}$ ). b No differences in IOP between sexes. There is a strong statistical association between the IOP of males and females $(P<0.0001)$. The relationship between the sexes is positively correlated $\left(R^{2}=0.37\right.$, Pearson correlation coefficient $\left.=0.62\right)$. c Genetic interval mapping revealed a single significant eQTL on proximal Chr 5. This is distinct from the locations of Tyrp1 and Gpnmb in the genome (black vertical lines on Chr 4 and Chr 6, respectively). d Tyrp1 and Gpnmb haplotypes do not influence IOP in BXD mice. The scatter plot shows average IOP of BXD strains carrying wild-type alleles of Tyrp1 and Gpnmb (WT/WT), wild-type allele of Tyrp1 and mutant allele of Gpnmb (MUT/WT), mutant allele of Tyrp1 and wildtype allele of Gpnmb (WT/MUT), and mutant alleles of both genes (MUT/MUT) ( $n=65, P=0.13$, age $=9.1-13$ months). $P$-value was calculated using an ANOVA. e Stringent selection criteria for selecting candidate genes. f A significant QTL for IOP is present on chromosome 5 between $14-19 \mathrm{Mb}$. Cacna2d1 is the strongest candidate gene located in the peak QTL. $\mathbf{g}$ Gene set enrichment analysis for positive correlates of Cacna2d1. Gene correlates of Cacna2d1 were significantly enriched for localization to the plasma membrane. False discovery rate (FDR) cutoff was set as $q \leq 0.25$. $\mathbf{h}$ Gene set enrichment analysis of genes positively correlated with Cacna2d1 presented as molecular function groupings. Categories that are statistically over-represented are shown with their normalized enrichment score (NES) listed next to the bars
} 
a

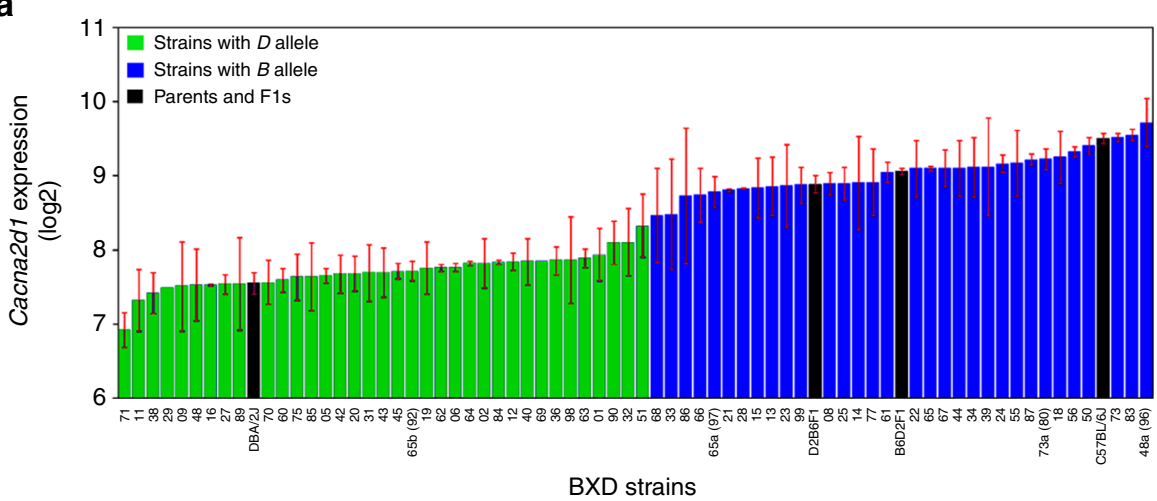

C

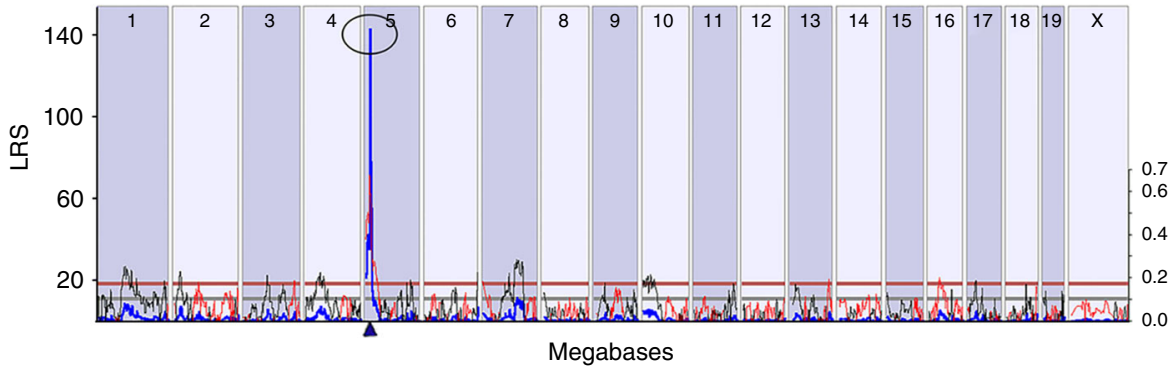

b

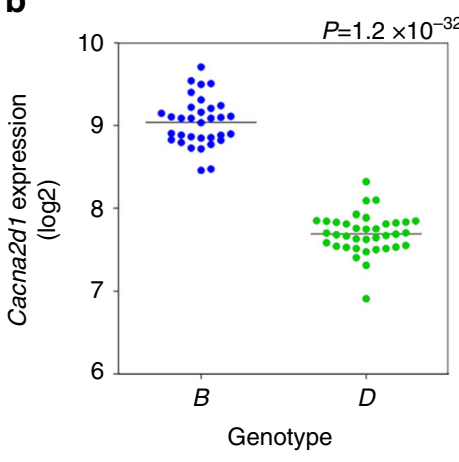

d

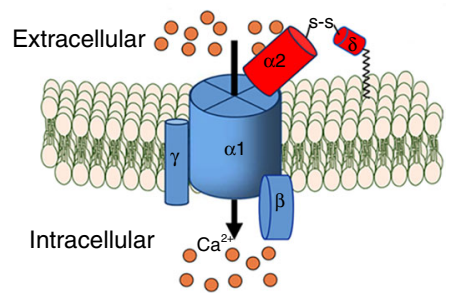

e

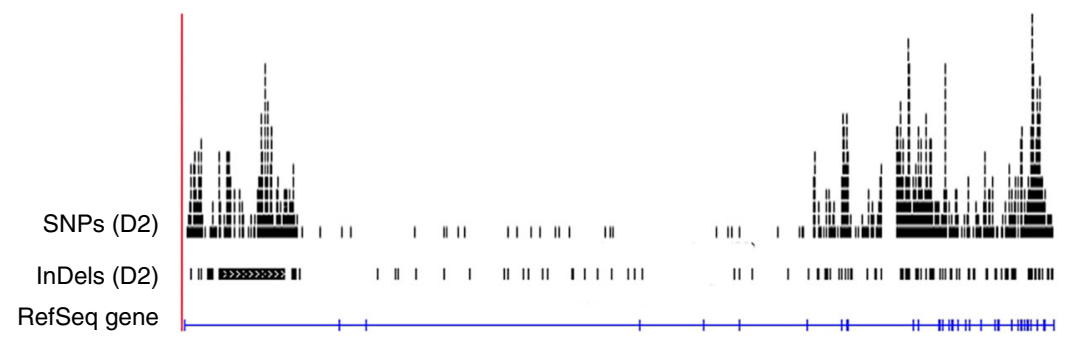

f

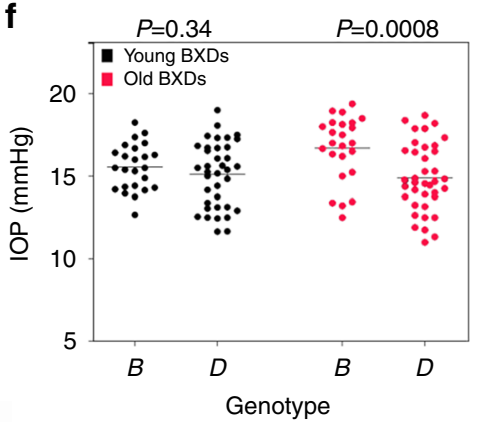

Fig. 3 Association analyses of Cacna2d1 haplotype variants. a Cacna2d1 expression in the whole eye varies across BXD strains. The bars depict a range of expression values from 6.9 \pm 0.03 and $9.7 \pm 0.10$ (mean \pm SEM). On the Y-axis, Cacna2d1 expression is on a log 2 scale. Parental strains (black), F1s (black), $D$ parental allele Cacna2d1 (green), and B parental allele of Cacna2d1 (red) are on X-axis. b Cacna2d1 haplotype influences Cacna2d1 expression level in eyes of BXD strains. BXD strains with the $D$ parental allele have lower expression of Cacna2d1 in the eye, while those with the $B$ parental allele have higher expression of the gene $(n=70 ; P<0.0001)$. $P$-value was calculated using an ANOVA. c Genetic mapping revealed a single highly significant cis-eQTL for Cacna2d1 on Chr 5. The purple triangle indicates the location of Cacna2d1 within the mouse genome. $\mathbf{d}$ Subunit assembly of voltage-gated calcium channels. Graphic representation of the high voltage-activated calcium channel complex consisting of the main pore forming $\alpha_{1}$ (blue) subunit plus ancillary, $\beta$ (blue), $\gamma$ (blue), and $\alpha_{2}$ (red) and $\delta_{1}$ (red) subunits. $\alpha_{2}$ and $\delta_{1}$ subunits have a disulphide bond between them. The $\delta_{1}$ unit is GPI-anchored to the plasma membrane. e UCSC Genome Browser illustration of gene structure, and location of SNPs and InDels in Cacna2d1 on Chr 5 of the mouse genome. Reference Sequence mRNA is represented in blue. $\mathbf{f}$ Effect of Cacna2d1 haplotype on IOP is age-dependent. In this scatter plot, parental allele of Cacna2d1 does not significantly influence IOP in young BXDs ( $P=0.34, n=63$, age $=1-2.1$ months; left). In contrast, BXD strains carrying the $B$ parental allele of Cacna2d1 have higher IOP, while those with the $D$ parental allele have lower IOP in older mice $(P=0.0008, n=64$, age $=9.1-13$ months; right). $P$-value was calculated using an ANOVA. F1s were not included in these analyses

significant association for these eight SNPs was not found in the IOP metadata, possibly due to different genetic effects in the multiple ethnicities included in that study.

CACNA2D1 is localized to the CB and TM in mice and humans. To further test Cacna2dl as a candidate IOPmodulating gene, we performed immunohistochemistry to determine the localization pattern of CACNA2D1 in healthy mouse and human donor eyes. In the mouse eye, CACNA2D1 is prominently localized to the TM, $\mathrm{CB}$, and ciliary muscle (CM) (Fig. 4a-c). CACNA2D1 was observed in a punctate pattern throughout the TM and Schlemm's canal. In the CB, CACNA2D1 was highly expressed in the non-pigmented epithelium. Weak labeling was present in the CM. A similar pattern of expression was observed in the TM and $\mathrm{CB}$ of the human donor eye (Fig. $4 \mathrm{~d}-\mathrm{f}$ ).

IOP response to pregabalin depends on Cacna2d1 genotype. Based upon our data suggesting that Cacna2dl modulates IOP, we evaluated the ability of pregabalin, a gabapentinoid drug with high specificity for CACNA2D1, to affect IOP. Our data demonstrate that pregabalin ophthalmic eye drops reduce IOP in mice in a dose-dependent manner (Fig. 5a). The percent reduction in IOP for both treated and control eyes after application of 


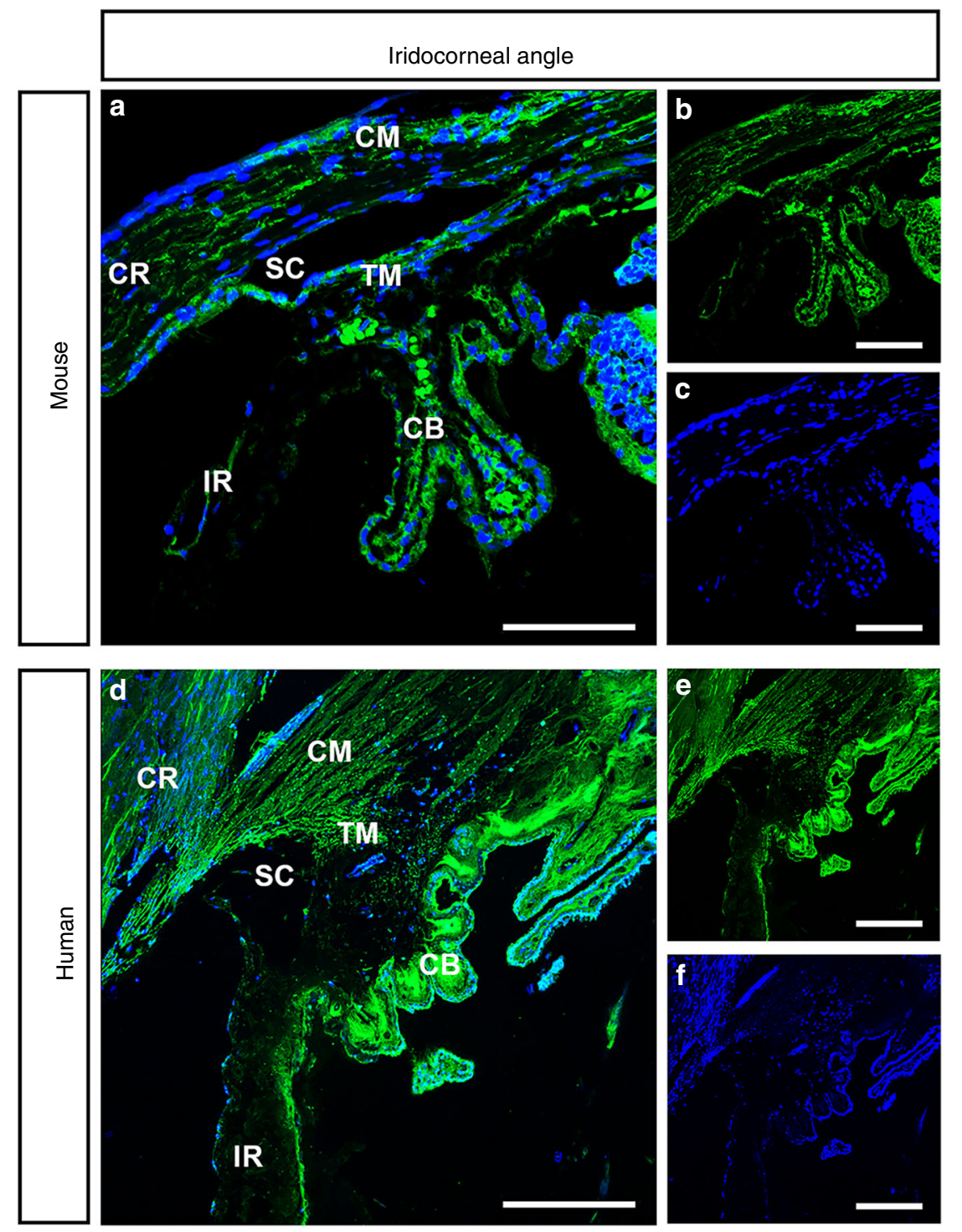

Fig. 4 Cellular localization of CACNA2D1 in C57BL/6 J mouse and human donor eyes. Cellular localization of CACNA2D1 in C57BL/6J mouse and human donor eyes. a-f Sections from C57BL/6J mouse (a-c) and human donor (d-f) iridocorneal angle were labeled with anti-CACNA2D1 antibodies. CACNA2D1 (green) is localized in the ciliary body (CB), trabecular meshwork (TM), Schlemm's canal (SC), and ciliary muscle (CM) in both B6 mice and human donor eyes. CACNA2D1 was also present in the posterior pigmented epithelium of the iris. $n=2$ mice and 1 human. Scale: $100 \mu$ m. Blue nuclei, CB ciliary body, CM ciliary muscle, CR cornea, IR iris, SC Schlemm's canal, TM trabecular meshwork

our ophthalmic pregabalin eye drops $(0.3-1.2 \%)$ are shown in Fig. 5a. Table 1 lists the pharmacodynamic parameters after application of pregabalin eye drops and statistical evaluation of the data, respectively. Drops containing $0.3 \%$ drug provided no IOP-lowering effect compared to control. All other concentrations of drug reduced IOP in a dose-dependent manner. A plateau was reached at $0.9 \%$ and there was no significant difference in drug response between 0.9 and $1.2 \%$ drug $(P>0.05)$. There was no significant difference between the time of maximum response $\left(T_{\max }\right)$ values of $0.6-1.2 \%$ concentrations of drug $(P>$ $0.05)$. In contrast, there was a significant difference between the time required for IOP to return to baseline $\left(T_{\text {end }}\right)$ for all concentrations of pregabalin eye drops, $(P<0.0001)$. The $1.2 \%$ pregabalin eye drops extended the duration of the IOP-lowering effect of pregabalin above that obtained with $0.6 \%$ pregabalin. Because there was no significant difference in the percent reduction of IOP between 0.9 and $1.2 \%$ pregabalin, we selected $0.9 \%$ as the minimal concentration required to produce the maximum reduction in IOP.
The Cacna2d1 haplotype also influenced the drug response. Figure $5 \mathrm{~b}$ illustrates the percent reduction in IOP after application of $0.9 \%$ pregabalin eye drops into the eyes of DBA/ 2J, BXD48 ( $D$ allele), C57BL/6J, and BXD14 ( $B$ allele) mice. Table 2 lists the pharmacodynamic parameters after application of $0.9 \%$ pregabalin eye drops and statistical evaluation of the data, respectively. Mice with the $B$ allele of Cacna2d1 (i.e., B6 and BXD14) were more responsive to pregabalin (0.9\%) eye drops than mice with the $D$ allele (i.e., D2 and BXD48). In addition to larger reductions in IOP, mice with the $B$ allele of Cacna2d 1 had larger $T_{\max }, T_{\text {end }}$, and area under the curve $\left(\mathrm{AUC}_{\mathrm{total}}\right)$ than mice with the $D$ allele. Expanding this analysis to an additional species, we observed a similar IOP-lowering response $(22.1 \pm$ $2.8 \%$ ) in Dutch belted rabbits after instillation of $0.9 \%$ pregabalin eye drops (Fig. 5c). Table 3 lists the pharmacodynamic parameters and statistical evaluation of the data after application of $0.9 \%$ pregabalin eye drops into the eyes of Dutch belted rabbits. 

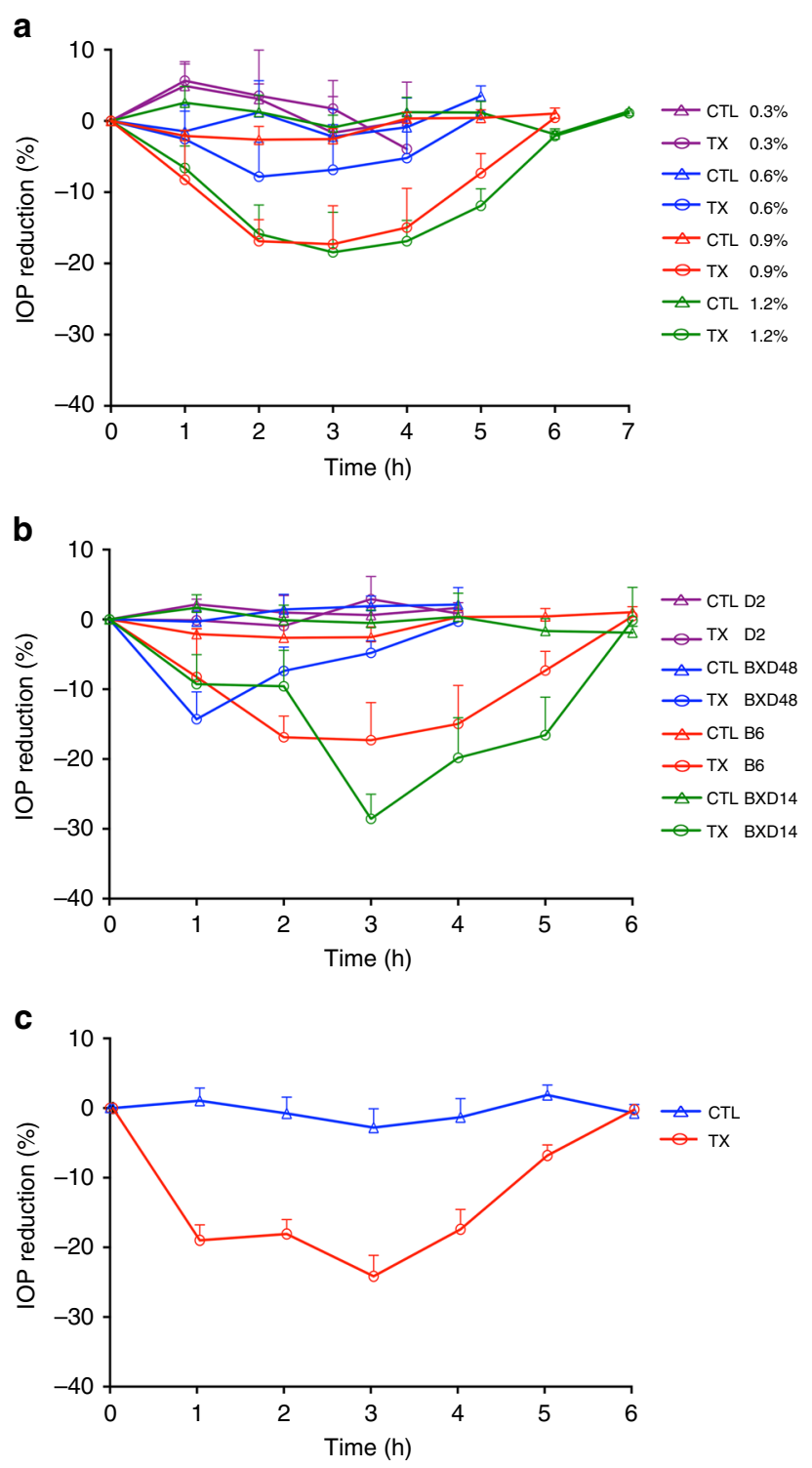

Fig. 5 Cacna2d1 haplotype effects IOP-lowering potency of pregabalin, a specific modulator of CACNA2D1, lowers IOP in B6 mice in a dosedependent manner. a We measured a dose-dependent reduction in IOP compared to control treatment after a single application of ophthalmic formulation containing a range of concentrations of pregabalin from 0.3 to $1.2 \%$. The minimal concentration required to produce the maximum reduction in IOP is $0.9 \%$ (mean $\pm \mathrm{SEM} ; n=6)$. Statistical details are provided in Table 1. b Pregabalin-induced IOP reduction is haplotypespecific. Mice carrying the $B$ parental allele of Cacna2d1 (i.e., B6 and BXD14) are more responsive to pregabalin $(0.9 \%)$ than mice carrying the $D$ parental allele (i.e., D2 and BXD48; mean \pm SEM; $n=4-6$ ). Statistical details are provided in Table 2. c A single dose of pregabalin eye drops (0.9\%) lowers IOP by $22.1 \%$ in Dutch belted rabbits. $n=5$. Statistical details are provided in Table 3

\section{Discussion}

By combining a forward murine genetics approach with cell biology, pharmacology, and analysis of human GWAS data, we were able to identify a genetic locus that significantly modulates IOP in the BXD murine genetic reference panel. Furthermore, we have demonstrated that our top candidate gene-Cacna2d1-is critical for modulation of IOP in these strains. Although other positional candidates in our Chr 5 QTL, such as Nos3 and Sema3e
(Supplementary Table 1), have been linked to glaucoma ${ }^{39-41}$, they do not fulfill our stringent selection criteria and therefore have been eliminated as candidate genes (Supplementary Table 2). Other positional candidates, such as Crygn and Klhl7, have been associated with other ocular diseases ${ }^{42}, 43$, but not glaucoma.

We have validated Cacna2d1 using multiple stringent criteria and identified human SNPs in the CACNA2D1 genomic region that are nominally associated with POAG. Furthermore, we have demonstrated that both baseline IOP and steady-state expression of Cacna2d1 are dependent upon the parental allele of the gene in mice. As a corollary, we further show that the response to pregabalin, a gabapentinoid drug with high affinity for CACNA2D1, is also dependent upon the Cacna2d1 haplotype. This is a bidirectional study that has successfully identified an IOP-modulating candidate gene in glaucoma and demonstrated a differential response to therapy. Our results further underscore the potential value of using the BXD genetic reference panel as a powerful tool to study human disease. Collectively, our data identify CACNA2D1 as a modulator of IOP and provide an avenue for a precision medicine approach to glaucoma therapy.

Previous studies have demonstrated a link between $\mathrm{Ca}^{2+}$, calcium channels and glaucoma ${ }^{44-53}$, providing a historical backdrop for our discovery of the role of Cacna2d1 in IOP modulation. Because $\mathrm{Ca}^{2+}$ likely plays a role in the pathophysiology of glaucoma ${ }^{45}$, systemic calcium channel blockers (CCBs) (e.g., verapamil, diltiazem, or nimodipine) have been evaluated as plausible therapies for POAG. However, the outcomes of these investigations have been inconsistent, with some studies demonstrating that CCBs are effective in lowering IOP, protecting ganglion cells, increasing ocular blood flow and improving visual function while others fail to replicate those results $^{46-53}$. In all studies performed to date, however, CCBs have targeted the $\alpha 1$ pore of the $\mathrm{Ca}^{2+}$ channel and have used a systemic route of administration. None of the previous investigations evaluated modulators of the $\mathrm{Ca}^{2+}$ channel auxiliary subunits, such as CACNA2D1, nor have they evaluated topical delivery of the drug. In our study, we demonstrate that targeting CACNA2D1 with topical pregabalin lowers IOP up to $30 \%$ in mice with the $B$ haplotype of the gene, which is a significantly higher effect than that demonstrated using systemically administered traditional CCBs.

Previous human genomic studies (GWAS and linkage studies) revealed multiple genomic regions that associated with elevated IOP and/or POAG ${ }^{7,9-11,16}$. Two regions of interest lie on Chr 7q near the locations of $C A C N A 2 D 1^{54-56}$ and $C A V 1 / C A V 2^{7}$. Other studies have demonstrated that SNPs in Cacna2d1/CACNA2D1 are associated with short QT syndrome ${ }^{38}$, perifosine cytotoxicity $^{57}$, altered sensitivity to acute noxious heat ${ }^{58}$, and beef quality traits ${ }^{59}$, indicating that polymorphisms in this gene have direct and distinct physiological consequences, some of which may be associated with modulation of IOP.

We show that CACNA2D1 is expressed in both CB and TM, the sites of $\mathrm{AH}$ production, and outflow through the traditional pathway, respectively, thus suggesting a possible role in $\mathrm{AH}$ dynamics. It is also modestly present in the CM, a structure that directly affects the distention of the TM in an antagonistic manner ${ }^{3}$. We also demonstrate that the Cacna2d1 likely functions in a network that includes other modulators of metal ion channel and transport activity. Based upon these collective data, we propose a model for the role of CACNA2D1 in regulating IOP (Fig. 6). Pregabalin, or other gabapentinoid drugs, binds to the CACNA2D1 subunit of the calcium channel, the affinity of which varies depending on the haplotype of the gene. The binding of the drug mitigates the flux of $\mathrm{Ca}^{2+}$ through the $\alpha 1$ pore of the calcium channel, reducing the level of intracellular $\mathrm{Ca}^{2+}$. A reduction in $\mathrm{Ca}^{2+}$ could cause a concomitant reduction in ion transport, water 
Table 1 Pharmacodynamic parameters after application of drops containing four concentrations of pregabalin to the eyes of B6 mice

Pharmacodynamic parameters (mean \pm SEM)

Pregabalin eye drops

Baseline IOP $(\mathrm{mmHg})$

IOP at $T_{\max }(\mathrm{mmHg})$

$\Delta \mathrm{IOP}(\mathrm{mmHg})$

$\%$ Reduction in IOP

$T_{\max }(\mathrm{h})$

$T_{\text {end }}(h)$

$A \cup C_{\text {total }}(\% h)$

\begin{tabular}{llll}
\hline $\mathbf{0 . 3} \%$ & $\mathbf{0 . 6 \%}$ & $\mathbf{0 . 9 \%}$ & $\mathbf{1 . 2 \%}$ \\
$15.4 \pm 0.3$ & $15.4 \pm 0.1$ & $15.3 \pm 0.1$ & $15.5 \pm 0.1$ \\
- & $14.2 \pm 0.7$ & $12.7 \pm 0.8$ & $12.6 \pm 0.8$ \\
- & $-1.2 \pm 0.8$ & $-2.7 \pm 0.8$ & $-2.9 \pm 0.9$ \\
- & $7.8 \pm 4.8$ & $17.3 \pm 5.4$ & $18.4 \pm 5.6$ \\
- & $3.0 \pm 0.4$ & $2.8 \pm 0.5$ & $3.4 \pm 0.4$ \\
- & $5.5 \pm 0.3$ & $6.0 \pm 0.4$ & $6.8 \pm 0.2$ \\
- & $42.9 \pm 11.1$ & $67.1 \pm 19.1$ & $71.9 \pm 12.1$
\end{tabular}

Statistical comparisons among four concentrations of pregabalin drops applied to the eyes of B6 mice

\begin{tabular}{|c|c|c|c|c|c|c|c|}
\hline $\begin{array}{l}\text { Pharmacodynamic parameters } \\
\text { (mean } \pm \text { SEM) }\end{array}$ & Overall $P$-value & $0.3 \%$ vs. $0.6 \%$ & $0.3 \%$ vs. $0.9 \%$ & $0.3 \%$ vs. $1.2 \%$ & $0.6 \%$ vs. $0.9 \%$ & $0.6 \%$ vs. $1.2 \%$ & $0.9 \%$ vs. $1.2 \%$ \\
\hline$\%$ Reduction in IOP & 0.034 & $>0.05$ & $>0.05$ & $<0.05$ & $>0.05$ & $>0.05$ & $>0.05$ \\
\hline$T_{\max }(\mathrm{h})$ & $<0.0001$ & $<0.001$ & $<0.001$ & $<0.0001$ & $>0.05$ & $>0.05$ & $>0.05$ \\
\hline$T_{\text {end }}(h)$ & $<0.0001$ & $<0.0001$ & $<0.0001$ & $<0.0001$ & $>0.05$ & $<0.05$ & $>0.05$ \\
\hline $\mathrm{AUC}_{\text {total }}(\% \mathrm{~h})$ & 0.002 & $>0.05$ & $<0.01$ & $<0.01$ & $>0.05$ & $>0.05$ & $>0.05$ \\
\hline
\end{tabular}

Overall $P$-value represents the outcome of the one-way ANOVA analysis. Individual $P$-values represent the outcome of Tukey-Kramer multiple comparisons tests. AUC $C_{\text {total }}(\% \mathrm{~h}$ ) area under the curve in percent IOP reduction $\mathrm{x}$ hours, $T_{\text {end }}(h)$ time to end of response in hours, $T_{\max }(h)$ time to maximum response in hours

Table 2 Pharmacodynamic parameters after application of $0.9 \%$ pregabalin eye drops to D2, BXD48 (D allele), B6, and BXD14 ( $B$ allele) mice

Pharmacodynamic parameters (mean \pm SEM)

Mouse strain

Baseline IOP $(\mathrm{mmHg})$

IOP at $T_{\max }(\mathrm{mmHg})$

$\triangle \mathrm{IOP}(\mathrm{mmHg})$

$\%$ Reduction in IOP

$T_{\max }(h)$

$T_{\text {end }}(h)$

$A \cup C_{\text {total }}(\% h)$

\begin{tabular}{llll}
\hline D2 & BXD48 & B6 & BXD14 \\
$17.2 \pm 0.3$ & $16.8 \pm 0.5$ & $15.3 \pm 0.1$ & $17.8 \pm 0.6$ \\
- & $14.4 \pm 0.7$ & $12.7 \pm 0.8$ & $12.7 \pm 0.5$ \\
- & $-2.4 \pm 0.6$ & $-2.7 \pm 0.8$ & $-5.1 \pm 0.8$ \\
- & $14.3 \pm 3.9$ & $17.3 \pm 5.4$ & $28.6 \pm 3.5$ \\
- & $1.5 \pm 0.2$ & $2.8 \pm 0.5$ & $3.2 \pm 0.3$ \\
- & $3.0 \pm 0.5$ & $6.0 \pm 0.4$ & $6.5 \pm 0.3$ \\
- & $26.6 \pm 4.5$ & $67.1 \pm 19.1$ & $88.7 \pm 12.6$ \\
\hline
\end{tabular}

Statistical comparisons among different strains of mice after application of $0.9 \%$ pregabalin eye drops

\begin{tabular}{llllllll}
\hline $\begin{array}{l}\text { Pharmacodynamic parameters } \\
\text { (mean } \pm \text { SEM) }\end{array}$ & Overall P-value & D2 vs. BXD48 & D2 vs. B6 & D2 vs. BXD14 & BXD48 vs. B6 & BXD48 vs. BXD14 & B6 vs. BXD14 \\
\hline$\%$ Reduction in IOP & 0.001 & $>0.05$ & $>0.05$ & $<0.001$ & $>0.05$ & $<0.05$ \\
$T_{\text {max }}(\mathrm{h})$ & $<0.0001$ & $<0.05$ & $<0.001$ & $<0.0001$ & $<0.05$ & $<0.01$ \\
$T_{\text {end }}(\mathrm{h})$ & $<0.0001$ & $<0.001$ & $<0.0001$ & $<0.0001$ & $<0.001$ & $<0.0001$ & $>0.05$ \\
AUC $_{\text {total }}(\% \mathrm{~h})$ & 0.0002 & $>0.05$ & $<0.01$ & $<0.001$ & $>0.05$ & $<0.01$ & $>0.05$ \\
\hline
\end{tabular}

Overall $P$-value represents the outcome of the one-way ANOVA analysis. Individual AUC-values represent the outcome of Tukey-Kramer multiple comparisons tests. AUC $\mathrm{C}_{\text {total }}(\% \mathrm{~h}$ ) area under the curve in percent IOP reduction $\mathrm{x}$ hours, $T_{\text {end }}(\mathrm{h})$ time to end of response in hours, $T_{\max }(\mathrm{h})$ time to maximum response in hours

Table 3 Pharmacodynamic parameters after application of $0.9 \%$ pregabalin eye drops to Dutch belted rabbits

\begin{tabular}{ll} 
Pharmacodynamic parameters & (Mean \pm SEM) \\
\hline Baseline IOP $(\mathrm{mmHg})$ & $20.7 \pm 0.5$ \\
IOP at $T_{\max }(\mathrm{mmHg})$ & $16.1 \pm 1.4$ \\
$\Delta I \mathrm{OP}(\mathrm{mmHg})$ & $-4.6 \pm 0.6$ \\
$\%$ Reduction in IOP & $22.1 \pm 2.8$ \\
$T_{\text {max }}(\mathrm{h})$ & $2.4 \pm 0.6$ \\
$T_{\text {end }}(\mathrm{h})$ & $6.0 \pm 0.0$ \\
$\mathrm{AUC} \mathrm{C}_{\text {total }}(\% \mathrm{~h})$ & $78.2 \pm 4.2$ \\
\hline AUC $_{\text {total }}(\% \mathrm{~h})$ area under the curve in percent IOP reduction $x$ hours, $T_{\text {end }}(\mathrm{h})$ time to end of \\
response in hours, $T_{\max }(\mathrm{h})$ time to maximum response in hours
\end{tabular}

transfer, and contraction of smooth muscle cells. The net result of pregabalin binding to CACNA2D1 could be threefold: a reduction in the production of $\mathrm{AH}$ by the $\mathrm{CB}$; a relaxation of the TM and resultant facilitation of the outflow of $\mathrm{AH}$; and relaxation of the CM, which would have an antagonistic effect on TM relaxation. Given the marked effect that pregabalin has on baseline IOP in BXD mice carrying the $B$ haplotype of Cacna2d1, we predict that the balance between the effect of the drug on TM cells and also CM cells would be in favor of increased outflow through the TM. If our hypothesis is valid, pregabalin binding to both $\mathrm{CB}$ and $\mathrm{TM}$, could both reduce $\mathrm{AH}$ production and increase AH outflow.

In summary, our study identified Cacna2d1 as a modifier of IOP. We offer a hypothesis regarding the modulation of IOP and 


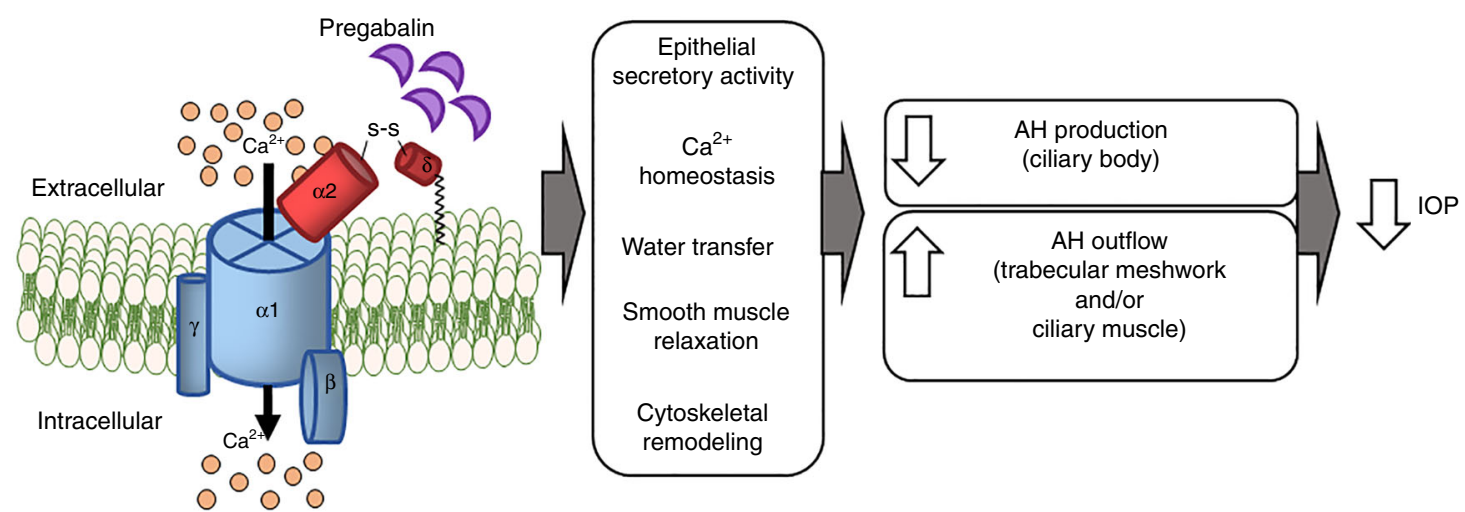

Fig. 6 Proposed mechanism of IOP reduction by pregabalin. We predict that pregabalin binds to CACNA2D1 localized to CB, TM, and/or CM cells. Pregabalin binding causes the $\alpha 1$ pore to close, leading to a decrease in $\mathrm{Ca}^{2+}$ influx into cells and a resultant decrease in free cytosolic $\mathrm{Ca}{ }^{2+}$. Consequently, the cells relax and aqueous humor inflow may be reduced and/or outflow may be increased, leading to a reduction in IOP

offer a potential therapeutic target that could preserve vision by reducing IOP. In the future, a similar approach could provide a bridge between systems biology and effective drug target identification.

\section{Methods}

Animals. A total of 65 BXD strains, parents, and F1 crosses (548 mice at 9.1-13 months age) were used for QTL mapping of IOP in mice aged 9.1-13 months. The gender distribution was relatively equal (313 females and 235 males). IOP measured from $64 \mathrm{BXD}$ strains aged 1-2.1 months (data available on GeneNetwork) were used for comparison. The parental strains C57BL/6J ( $n=6$; aged 3-5 months), DBA/2J ( $n=4$; aged 3-5 months), and two BXD strainsBXD14 and BXD48 ( $n=6$; aged 3-5 months) - were used to evaluate the IOPlowering effects of pregabalin. C57BL/6 J and DBA/2 J were used as controls. BXD14 and BXD48 strains were selected based on presence of the $B$ and $D$ haplotype of Cacna2d1, respectively. Dutch belted rabbits $(n=5$; procured from Robinson Services, Mocksville, NC, USA) weighing 2.0-2.5 kg were used to test IOP-lowering effects of pregabalin. All procedures involving mice and rabbits were approved by the Animal Care and Use review board of the University of Tennessee Health Science Center (UTHSC) and followed the Association of Research in Vision and Ophthalmology Statement for the Use of Animals in Ophthalmic and Vision Research in addition to the guidelines for laboratory animal experiments (Institute of Laboratory Animal Resources, Public Health Service Policy on Humane Care and Use of Laboratory Animals). Animals were housed under cyclic light ( $12 \mathrm{~h}$ on: $12 \mathrm{~h}$ off) with $35 \%$ humidity in a specific pathogen-free facility at UTHSC, and were allowed free access to water and food.

Human donor tissue. Our research and the protocol for collection of human retinas were conducted in accordance with the tenets of the Declaration of Helsinki and approved by University of Tennessee Health Science Center Institutional Review Board. Informed consent was received from donors or donor family members. Paraffin sections from a human donor eye (70-year-old, male) with no diagnosis of ocular disease were obtained from National Disease Research Interchange (Philadelphia, PA). No donor details apart from age, sex, and cause of death were provided.

IOP measurement. IOP of mice was measured using an induction-impact tonometer (Tonolab tonometer, Colonial Medical Supply, Franconia, NH) for rodents according to the manufacturer's recommended procedures. The IOP of rabbits was measured using a Tono-Pen AVIA (Reichert Technologies, Depew, NY) while rabbits were held in a rabbit restrainer. Six consecutive IOP readings were averaged. For each mouse, the tested ophthalmic formulation $(10 \mu \mathrm{l})$ was applied to the right eye, while the left eye received the blank and serve as a control ${ }^{29,60}$. One hundred microliters of $0.9 \%$ pregabalin eye drops were applied into the inferior conjunctival sac of the right eye of the Dutch belted rabbits, while the left eye received the blank eye drops ${ }^{61}$. All results were expressed as the mean percentage reduction in IOP from baseline (mean\% reduction \pm SEM).

Identification of IOP-modulating loci in mice. IOP data generated from 9.1-13month-old BXD strains was integrated into the GeneNetwork database. The identification and mapping of phenotypic QTL was performed by linking trait data to genotypes at known genetic marker loci using the WebQTL module on GeneNetwork (http://www.genenetwork.org/) 26, 29, 30, 32 .
Evaluation of CACNA2D1 in GWAS repositories. SNPs located within the CACNA2D1 human genomic region were evaluated in the NEIGHBORHOOD consortium metadata ${ }^{16}$. To capture regulatory regions, the genomic locus was defined as all coding sequences +50 base pairs on either side of the first/last exon.

eQTL analysis of IOP candidate genes. IOP data from BXD mice aged 1-2.1 months (64 strains) and 9.1-13 months (65 strains) are publically available as BXD published phenotypes record IDs 16337 (http://www.gn2.genenetwork.org/ show_trait?trait_id=16337\&dataset=BXDPublish) and 16340 (http://www.gn2. genenetwork.org/show_trait?trait_id=16340\&dataset=BXDPublish), respectively. Candidate genes were identified using the tools available on the GeneNetwork/ UCSC Genome browser. Gene expression was used as a microtrait to map regulatory eQTLs for the differences in mRNA expression levels over the panel of $\mathrm{BXD}$ lines because it is a heritable trait. Whole-eye transcript data from BXD strains available on GeneNetwork as Eye M430v2 (Sep08) RMA (http://www. genenetwork.org/webqtl/main.py?FormID=sharinginfo\&GN_AccessionId=207) were used to identify cis (locally)-regulated genes from within intervals of inter$\mathrm{est}^{34}$. The same transcript data were used to identify genes whose expression correlated with IOP at 9.1-13 months of age. Only probes that did not bind to regions containing SNPs were used to avoid hybridization artifacts that may cause differences in expression due to technical error rather than biological variance. All probes were verified by BLAST-like alignment tool (BLAT) (University of California Santa Cruz (UCSC) Genome Browser). Correlation analyses for initial candidate gene selection used Pearson correlation coefficients. Genes were considered as being cis-regulated if the associated marker was localized within a $10 \mathrm{Mb}$ distance of the gene position ${ }^{34}$.

Gene set enrichment analysis. GSEA was performed as described previously using version 2.2.0 (http://www.broadinstitute.org/gsea ${ }^{35,62}$ ). Briefly, all nominally significant correlates (uncorrected $P \leq 0.05,1603$ genes) of Cacna2d1 were extracted from the whole-eye transcript data and pre-ranked based on their correlation coefficients (Supplementary Data 1). Molecular Signatures Database (MSigDB) version 5.0 was used as the database to perform GSEA. GSEA was performed on these pre-ranked genes using 1000 permutations. This was followed by building modules of related pathways based on at least $25 \%$ gene overlap between pathways using the enrichment map strategy. The enrichment score (ES) was calculated as cumulative score from 0 . The ES was normalized to account for the size of the gene set being used. To control the expected proportions of false positives, the FDR for $P$-values was calculated using the Benjamini and Hochberg method implemented in LIMMA.

Immunohistochemistry. Our standard methods were used to immunolocalize CACNA2D1 in mouse and human eye sections ${ }^{30,63,64}$. An anti-CACNA2D1 antibody (Bioss Antibodies, Woburn, MA; catalog \#bs-11981R; 1:100) was used in these studies along with goat anti-rabbit Alexa fluor 488 secondary antibody (Invitrogen, Waltham, MA; catalog \#A11034; 1:200), and TO-PRO-3 iodide (Invitrogen, Waltham, MA; catalog \#T3605) as a nuclear counterstain. Sections were viewed and images were obtained using a Nikon $\mathrm{C} 1$ confocal microscope (Nikon, NY).

Topical pregabalin ophthalmic formulations. Blank ophthalmic eye drops were prepared by dissolving $2 \%$ hydroxypropylmethylcellulose (Sigma-Aldrich, $\mathrm{St}$. Louis, MO) in PBS pH 7.4 to use it as a vehicle (80\%) in combination with propylene glycol (10\%; Sigma-Aldrich) and PEG 300 (10\%; Sigma-Aldrich). Pregabalin (Sigma-Aldrich, St. Louis, MO) ophthalmic eye drops were prepared 
using four different concentrations of the drug $(0.3,0.6,0.9$, and $1.2 \%)$ by dissolving pregabalin in the previously prepared blank ophthalmic eye drops. These studies were conducted using a single dose-response design. Ten microliters of the pregabalin formulations or the blank ophthalmic eye drops were used in the mouse studies $^{60}(n=4-6)$. One hundred microliters of either formulation were used in the rabbit study $(n=5)$. IOP was measured prior to treatment and hourly until the IOP returned to baseline levels.

Several pharmacodynamics parameters were used to evaluate pregabalin including maximum reduction in IOP (\% reduction in IOP), time required to reach maximum decrease of IOP $\left(T_{\max }\right)$, time required for IOP to return to baseline (i.e., end of drug effect; $T_{\text {end }}$ ), and total area under the percent reduction in IOP-vs-time curve $\left(\mathrm{AUC}_{\text {total }}\right)$. Values of $\mathrm{AUC}_{\text {total }}$ were determined using a linear trapezoidal method $^{65}$. Results were reported as mean \pm SEM.

Statistics. To determine how much of the variation in candidate gene across the cohort was due to genetic effects, we calculated the heritability of the candidate gene using the formula of Hegmann and Possidente ${ }^{26,32,33}$. Pearson productmoment correlations were calculated using the Correlation matrix tool available in GeneNetwork. Pearson correlation, denoted by $r$, measures the strength of a linear association between two variables, which in our case are IOP $(\mathrm{mmHg})$ and $\mathrm{Cac}$ na2d 1 mRNA expression. Partial correlation within-strain and between-strain variances, genotypes vs. IOP, and genotype vs. gene expression were calculated with analysis of variance (ANOVA) using SAS 6.0. (Cary, NC) or GraphPad Prism7 software (La Jolla, CA). Statistical analyses of the pharmacodynamics results for pregabalin were performed using one-way ANOVA with Tukey-Kramer multiple comparisons test. All pharmacodynamic parameters as well as the statistical analysis of the results were also calculated using GraphPad Prism-7 software (La Jolla, CA).

Data availability. The data sets generated during and/or analyzed during the current study are available in the GeneNetwork repository as follows: IOP data from BXD mice aged 1-2.1 months and 9.1-13 months are publically available as BXD published phenotypes record IDs 16337 (http://www.gn2.genenetwork.org/ show_trait?trait_id=16337\&dataset=BXDPublish) and 16340, (http://www.gn2. genenetwork.org/show_trait?trait_id=16340\&dataset=BXDPublish), respectively. Cacna2d1 expression data is available as Trait ID 1446324_at and 1449999_a_at in the Eye M430v2 (Sep08) RMA database (http://www.genenetwork.org/webqtl/ main.py?FormID=sharinginfo\&GN_AccessionId=207).

Received: 1 December 2016 Accepted: 28 June 2017

Published online: 24 November 2017

\section{References}

1. Kwon, Y. H., Fingert, J. H., Kuehn, M. H. \& Alward, W. L. M. Primary openangle glaucoma. N. Engl. J. Med. 360, 1113-1124 (2009).

2. Goel, M., Picciani, R. G., Lee, R. K. \& Bhattacharya, S. K. Aqueous humor dynamics: a review. Open Ophthalmol. J. 4, 52-9 (2010).

3. Tamm, E. The trabecular meshwork outflow pathways: structural and functional aspects. Exp. Eye. Res. 88, 648-655 (2009).

4. Llobet, A., Gasull, X. \& Gual, A. Understanding trabecular meshwork physiology: a key to the control of intraocular pressure? News Physiol. Sci. 18, 205-9 (2003).

5. van Koolwijk, L. M. et al. Genetic contributions to glaucoma: heritability of intraocular pressure, retinal nerve fiber layer thickness, and optic disc morphology. Invest. Ophthalmol. Vis. Sci. 48, 3669-76 (2007).

6. Carbonaro, F., Andrew, T., Mackey, D. A., Spector, T. D. \& Hammond, C. J. Heritability of intraocular pressure: a classical twin study. Br. J. Ophthalmol. 92, 1125-8 (2008).

7. Thorleifsson, G. et al. Common variants near CAV1 and CAV2 are associated with primary open-angle glaucoma. Nat. Genet. 42, 906-9 (2010).

8. Wiggs, J. et al. Common variants at $9 \mathrm{p} 21$ and $8 \mathrm{q} 22$ are associated with increased susceptibility to optic nerve degeneration in glaucoma. PLoS Genet. 8 , e1002654 (2012).

9. Hysi, P. et al. Genome-wide analysis of multi-ancestry cohorts identifies new loci influencing intraocular pressure and susceptibility to glaucoma. Nat. Genet. 46, 1126-1130 (2014).

10. Gharahkhani, P. et al. Common variants near ABCA1, AFAP1 and GMDS confer risk of primary open-angle glaucoma. Nat. Genet. 46, 1120-1125 (2014).

11. Burdon, K. et al. Genome-wide association study identifies susceptibility loci for open angle glaucoma at TMCO1 and CDKN2B-AS1. Nat. Genet. 46, 574-578 (2011).

12. Chen, Y. et al. Common variants near ABCA1 and in PMM2 are associated with primary open-angle glaucoma. Nat. Genet. 46, 1115-1119 (2014).
13. Nag, A. et al. A genome-wide association study of intra-ocular pressure suggests a novel association in the gene FAM125B in the TwinsUK cohort. Hum. Mol. Genet. 23, 3343-3348 (2014).

14. $\mathrm{Li}, \mathrm{Z}$. et al. A common variant near TGFBR3 is associated with primary open angle glaucoma. Hum. Mol. Genet. 24, 3880-3892 (2015).

15. Springelkamp, H. et al. ARHGEF12 influences the risk of glaucoma by increasing intraocular pressure. Hum. Mol. Genet. 24, 2689-2699 (2015).

16. Bailey, J. N. et al. Genome-wide association analysis identifies TXNRD2, ATXN2 and FOXC1 as susceptibility loci for primary open-angle glaucoma. Nat. Genet. 48, 189-94 (2016).

17. Wang, X. et al. Joint mouse-human phenome-wide association to test gene function and disease risk. Nat. Commun. 7, 10464 (2016).

18. Houtkooper, R. H. et al. Mitonuclear protein imbalance as a conserved longevity mechanism. Nature 497, 451-7 (2013).

19. Hager, R., Lu, L., Rosen, G. D. \& Williams, R. W. Genetic architecture supports mosaic brain evolution and independent brain-body size regulation. Nat. Commun. 3, 1079 (2012).

20. Andreux, P. A. et al. Systems genetics of metabolism: the use of the BXD murine reference panel for multiscalar integration of traits. Cell 150, 1287-99 (2012).

21. Peirce, J. L., Lu, L., Gu, J., Silver, L. M. \& Williams, R. W. A new set of BXD recombinant inbred lines from advanced intercross populations in mice. $B M C$ Genet. 5, 7 (2004).

22. Taylor, B. A. et al. Genotyping new BXD recombinant inbred mouse strains and comparison of BXD and consensus maps. Mamm. Genome 10, 335-48 (1999).

23. Chesler, E. J. et al. Complex trait analysis of gene expression uncovers polygenic and pleiotropic networks that modulate nervous system function. Nat. Genet. 37, 233-42 (2005).

24. Ashbrook, D. G., Gini, B. \& Hager, R. Genetic variation in offspring indirectly influences the quality of maternal behaviour in mice. eLife 4, el1814 (2015).

25. Williams, E. G. et al. Systems proteomics of liver mitochondria function. Science 352, aad0189 (2016).

26. Lu, H. et al. Complex interactions of Tyrp1 in the eye. Mol. Vis. 17, 2455-2468 (2011).

27. Jablonski, M. M. et al. Genetic pathways regulating glutamate levels in retinal Muller cells. Neurochem. Res. 36, 594-603 (2011).

28. Templeton, J. P. et al. Innate immune network in the retina activated by optic nerve crush. Invest. Ophthalmol. Vis. Sci. 54, 2599-606 (2013).

29. Lu, H., Lu, L., Williams, R. \& Jablonski, M. Iris transillumination defect and its gene modulators do not correlate with intraocular pressure in the BXD family of mice. Mol. Vis. 22, 224-233 (2016).

30. Chintalapudi, S. R., Morales-Tirado, V. M., Williams, R. W. \& Jablonski, M. M. Multipronged approach to identify and validate a novel upstream regulator of Sncg in mouse retinal ganglion cells. FEBS J. 283, 678-93 (2016).

31. Anderson, M. G. et al. Mutations in genes encoding melanosomal proteins cause pigmentary glaucoma in DBA/2J mice. Nat. Genet. 30, 81-5 (2002).

32. Lu, H. et al. Genetic dissection of the Gpnmb network in the eye. Invest. Ophthalmol. Vis. Sci. 52, 4132-42 (2011).

33. Swaminathan, S., Lu, H., Williams, R. W., Lu, L. \& Jablonski, M. M. Genetic modulation of the iris transillumination defect: a systems genetics analysis using the expanded family of BXD glaucoma strains. Pigment Cell Melanoma Res. 26, 487-98 (2013).

34. Freeman, N. E. et al. Genetic networks in the mouse retina: growth associated protein 43 and phosphatase tensin homolog network. Mol. Vis. 17, 1355-72 (2011).

35. Subramanian, A. et al. Gene set enrichment analysis: a knowledge-based approach for interpreting genome-wide expression profiles. Proc. Natl Acad. Sci. USA 102, 15545-50 (2005).

36. Schleithoff, L., Mehrke, G., Reutlinger, B. \& Lehmann-Horn, F. Genomic structure and functional expression of a human alpha(2)/delta calcium channel subunit gene (CACNA2). Genomics 61, 201-9 (1999).

37. Risgaard, B. et al. High prevalence of genetic variants previously associated with Brugada syndrome in new exome data. Clin. Genet. 84, 489-95 (2013).

38. Templin, C. et al. Identification of a novel loss-of-function calcium channel gene mutation in short QT syndrome (SQTS6). Eur. Heart J. 32, 1077-1088 (2011).

39. Lei, Y., Zhang, X., Song, M., Wu, J. \& Sun, X. Aqueous Humor Outflow Physiology in NOS3 Knockout Mice. Invest. Ophthalmol. Vis. Sci. 56, 4891-8 (2015).

40. Kang, J. H., Wiggs, J. L., Haines, J., Abdrabou, W. \& Pasquale, L. R. Reproductive factors and NOS3 variant interactions in primary open-angle glaucoma. Mol. Vis. 17, 2544-51 (2011).

41. Smit-McBride, Z. et al. In vivo gene expression profiling of retina postintravitreal injections of dexamethasone and triamcinolone at clinically relevant time points for patient care. Invest. Ophthalmol. Vis. Sci. 52, 8965-78 (2011). 
42. Hugosson, T. et al. Phenotype associated with mutation in the recently identified autosomal dominant retinitis pigmentosa KLHL7 gene. Arch. Ophthalmol. 128, 772-8 (2010).

43. Reis, L. M. et al. Whole exome sequencing in dominant cataract identifies a new causative factor, CRYBA2, and a variety of novel alleles in known genes. Hum. Genet. 132, 761-770 (2013).

44. McElnea, E. M. et al. Oxidative stress, mitochondrial dysfunction and calcium overload in human lamina cribrosa cells from glaucoma donors. Mol. Vis. 17, 1182-1191 (2011).

45. Crish, S. \& Calkins, D. Neurodegeneration in glaucoma: progression and calcium-dependent intracellular mechanisms. Neuroscience 176, 1-11 (2011).

46. Payne, L., Slagle, T., Cheeks, L. \& Green, K. Effect of calcium channel blockers on intraocular pressure in rabbits. Ophthalmic Res. 22, 337-341 (1990).

47. Wiederholt, M., Thieme, H. \& Stumpff, F. The regulation of trabecular meshwork and ciliary muscle contractility. Prog. Retin. Eye Res. 19, 271-295 (2000).

48. Siegner, S., Netland, P., Schroeder, A. \& Erickson, K. Effect of calcium channel blockers alone and in combination with antiglaucoma medications on intraocular pressure in the primate eye. J. Glaucoma 9, 334-339 (2000).

49. Luksch, A. et al. Effect of nimodipine on ocula rblood flow and colour contrast sensitivity in patients with normal tension glaucoma. Br. J. Ophthalmol. 89, 21-25 (2005).

50. Jani, A., Goyal, R., Shah, G. \& Mehta, A. Effect of calcium channel blockers on intraocular pressure in rabbits. Iranian J. Pharmacol. Ther. 4, 95-99 (2005).

51. Araie, M. \& Mayama, C. Use of calcium channel blockers for glaucoma. Prog. Retin. Eye Res. 30, 54-71 (2011).

52. Mayama, C. Calcium channels and their blockers in intraocular pressure and glaucoma. European J Pharm 739, 96-105 (2014).

53. Ganekal, S., Dorairaj, S., Jhanji, V. \& Kudlu, K. Effect of topical calcium channel blockers on intraocular pressure in steroid-induced glaucoma. J. Curr. Glaucoma Pract. 8, 15-19 (2014).

54. Andersen, J. S. et al. A gene responsible for the pigment dispersion syndrome maps to chromosome 7q35-q36. Arch. Ophthalmol. 115, 384-8 (1997).

55. Wirtz, M. K. et al. GLC1F, a new primary open-angle glaucoma locus, maps to 7q35-q36. Arch. Ophthalmol. 117, 237-41 (1999).

56. Klein, B. E. et al. Prevalence of glaucoma. The Beaver Dam Eye Study. Ophthalmology 99, 1499-504 (1992).

57. Zhang, W., Liu, W., Poradosu, E. \& Ratain, M. Genome-wide identification of genetic determinants for the cytotoxicity of perifosine. Hum. Genomics 3, 53-70 (2008).

58. Neely, G. et al. A genome-wide Drosophila screen for heat nociception identifies $\alpha 2 \delta 3$ as an evolutionarily conserved pain gene. Cell 143, 628-638 (2010).

59. Yuan, Z. \& Xu, S. Novel SNPs of the bovine CACNA2D1 gene and their association with carcass and meat quality traits. Mol. Biol. Rep. 38, 365-370 (2011).

60. Ibrahim, M., Abd-Elgawad, A., Soliman, O. \& Jablonski, M. Natural bioadhesive biodegradable nanoparticle-based topical ophthalmic formulations for management of glaucoma. Transl. Vis. Sci. Technol. 4, 12 (2015).

61. Swaminathan, S. et al. Novel endogenous glycan therapy for retinal diseases: safety, in vitro stability, ocular pharmacokinetic modeling and bio-distribution. AAPS J. 16, 311-312 (2014).

62. Mootha, V. K. et al. PGC-1alpha-responsive genes involved in oxidative phosphorylation are coordinately downregulated in human diabetes. Nat. Genet. 34, 267-73 (2003).

63. Nookala, S. et al. In search of the identity of the XAP-1 antigen: a protein localized to cone outer segments. Invest. Ophthalmol. Vis. Sci. 51, 2736-43 (2010).
64. Chintalapudi, S. R. et al. Isolation and molecular profiling of primary mouse retinal ganglion cells: comparison of phenotypes from healthy and glaucomatous retinas. Front. Aging Neurosci. 8, 93 (2016).

65. Aburahma, M. H. \& Mahmoud, A. A. Biodegradable ocular inserts for sustained delivery of brimonidine tartarate: preparation and in vitro/in vivo evaluation. AAPS PharmSciTech. 12, 1335-47 (2011).

\section{Acknowledgements}

We thank Dr. E. Geisert, Dr. L. Lu, and Mr. B. Orr for their assistance in generating the BXD microarray data sets that were used in these analyses. We also thank Dr. H. Lu for acquiring the IOP data sets and Dr. S. Surbhi for assistance with statistical analyses. We further thank S. Ganguli and A. Shepherd for discussion and technical assistance. We acknowledge the financial support from: the Center for Integrative and Translational Genomics at the University of Tennessee Health Science Center; NEI Grants R01EY021200, R01EY022305, and P30EY013080; NIAAA Grant U01AA01666; and a Stein Innovation Award and an unrestricted grant from Research to Prevent Blindness, Inc.

\section{Author contributions}

S.R.C. and M.M.J. conceived the experiments. S.R.C., D.M., and X.W. conducted experiments. S.R.C., D.M., X.W., P.G.H., J.N.C.B., R.W.W., J.L.W., and M.M.J. participated in data interpretation and discussion. M.M.J. conceptualized the project and supervised the experiments. R.W.W. and M.M.J. provided resources/materials/analysis tools for completion of the study. S.R.C. and M.M.J. wrote the manuscript. All authors reviewed and contributed intellectually to the article.

\section{Additional information}

Supplementary Information accompanies this paper at doi:10.1038/s41467-017-00837-5

Competing interests: The authors declare no competing financial interests.

Reprints and permission information is available online at http://npg.nature.com/ reprintsandpermissions/

Publisher's note: Springer Nature remains neutral with regard to jurisdictional claims in published maps and institutional affiliations.

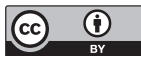

Open Access This article is licensed under a Creative Commons Attribution 4.0 International License, which permits use, sharing, adaptation, distribution and reproduction in any medium or format, as long as you give appropriate credit to the original author(s) and the source, provide a link to the Creative Commons license, and indicate if changes were made. The images or other third party material in this article are included in the article's Creative Commons license, unles indicated otherwise in a credit line to the material. If material is not included in the article's Creative Commons license and your intended use is not permitted by statutory regulation or exceeds the permitted use, you will need to obtain permission directly from the copyright holder. To view a copy of this license, visit http://creativecommons.org/ licenses/by/4.0/

(C) The Author(s) 2017

\section{NEIGHBORHOOD consortium}

Rand Allingham ${ }^{9}$, Murray Brilliant ${ }^{10}$, Don Budenz ${ }^{11}$, John Fingert ${ }^{12}$, Douglas Gaasterland ${ }^{13}$, Teresa Gaasterland $^{13}$, Jonathan L. Haines ${ }^{4}$, Lisa Hark ${ }^{14}$, Michael Hauser ${ }^{9}$, Rob Igo ${ }^{4}$, Jae Hee Kang ${ }^{15}$, Peter Kraft ${ }^{16}$, Richard Lee ${ }^{17}$, Paul Lichter ${ }^{18}$, Yutao Liu ${ }^{19}$, Syoko Moroi ${ }^{18}$, Louis R. Pasquale ${ }^{6,15}$, Margaret Pericak-Vance ${ }^{20}$, Anthony Realini ${ }^{21}$, Doug Rhee ${ }^{22}$, Julia R. Richards ${ }^{18}$, Robert Ritch ${ }^{23}$, Joel Schuman ${ }^{24}$, William K. Scott ${ }^{25}$, Kuldev Singh ${ }^{26}$, Arthur Sit ${ }^{27}$, Douglas Vollrath ${ }^{28}$, Gadi Wollstein ${ }^{24}$ \& Don Zack ${ }^{29}$

${ }^{9}$ Department of Ophthalmology, Duke University Eye Center, Durham, NC 27705, USA. ${ }^{10}$ University of Wisconsin Institute for Clinical and Translational Research, Madison, WI 53705, USA. ${ }^{11}$ Department of Ophthalmology, UNC School of Medicine, Chapel Hill, NC 27517, USA.

${ }^{12}$ Department of Ophthalmology and Visual Sciences, University of lowa, lowa City, IA 52242, USA. ${ }^{13}$ Eye Doctors of Washington DC, Washington, 
DC 20036, USA. ${ }^{14}$ Glaucoma Research Center, Wills Eye Hospital, Philadelphia, PA 19107, USA. ${ }^{15}$ Channing Division of Network Medicine, Brigham and Women's Hospital, Boston, MA 02115, USA. ${ }^{16}$ Harvard T.H. Chan School of Public Health, Boston, MA 02115, USA. ${ }^{17}$ Department of Ophthalmology, Bascom Palmer Eye Institute, University of Miami, Miami, FL 33136, USA. ${ }^{18}$ Department of Ophthalmology, Kellogg Eye Center, University of Michigan, Ann Arbor, MI 48105, USA. ${ }^{19}$ Department of Cellular Biology and Anatomy, Augusta University, Augusta, GA 30901, USA. ${ }^{20}$ John P. Hussman Institute for Human Genomics, University of Miami, Miami, FL 33136, USA. ${ }^{21}$ Department of Ophthalmology, West Virginia University, Morgantown, WV 26506, USA. ${ }^{22}$ Department of Ophthalmology and Visual Sciences, Case Western Reserve University, Cleveland, OH 44106, USA. ${ }^{23}$ Department of Ophthalmology, Einhorn Clinical Research Center, New York Eye and Ear Infirmary, New York, NY 10003, USA.

${ }^{24}$ Department of Ophthalmology, NYU Langone Medical Center, New York, NY 10016, USA. ${ }^{25}$ Dr. John T. Macdonald Foundation Department of Human Genetics, John P. Hussman Institute for Human Genomics, University of Miami Health System, Miami, FL 33136, USA. ${ }^{26}$ Department of Ophthalmology, Stanford University Medical Center, Stanford, CA 94303, USA. ${ }^{77}$ Department of Ophthalmology, Mayo Clinic, Rochester, MN 85259, USA. ${ }^{28}$ Department of Genetics, Stanford University Medical Center, Stanford, CA 94305, USA. ${ }^{29}$ Department of Ophthalmology, Wilmer Eye Institute, The Johns Hopkins University School of Medicine, Baltimore, MD 21287, USA

\section{International Glaucoma Genetics consortium}

Tin Aung ${ }^{30}$, Peter Bonnemaijer ${ }^{31}$, Cheng-Yu Cheng ${ }^{31}$, Jamie Craig ${ }^{32}$, Cornelia van Duijn ${ }^{33}$, Puya Gharahkhani ${ }^{34}$, Adriana Iglesias Gonzalez ${ }^{35}$, Christopher J. Hammond ${ }^{36}$, Alex Hewitt ${ }^{37}$, Rene Hoehn ${ }^{38}$, Fridbert Jonansson ${ }^{39}$, Anthony Khawaja ${ }^{40}$, Chiea Chuen Khor ${ }^{41}$, Caroline C.W. Klaver ${ }^{42}$, Andrew Lotery ${ }^{43}$, David Mackey ${ }^{44}$, Stuart MacGregor ${ }^{45}$, Calvin Pang ${ }^{46}$, Francesca Pasutto ${ }^{47}$, Kári Stefansson ${ }^{48}$, Gudmar Thorleifsson ${ }^{48}$, Unnar Thorsteinsdottir ${ }^{48}$, Veronique Vitart ${ }^{49}$, Eranga Vithana ${ }^{30}$, Terri Young ${ }^{50} \&$ Tanja Zeller ${ }^{51}$

${ }^{30}$ Singapore Eye Research Institute, National University of Singapore, Singapore, 168751, Singapore. ${ }^{31}$ Department of Epidemiology, Erasmus Medical Center, Rotterdam, GE 3015, The Netherlands. ${ }^{32}$ Department of Ophthalmology, Flinders University, Adelaide, SA 5042, Australia. ${ }^{33}$ Department of Epidemiology, Erasmus Medical Center, Rotterdam, GE 3015, The Netherlands. ${ }^{34}$ Statistical Genetics, QIMR Berghofer Medical Research Institute, Brisbane, QLD 4006, Australia. ${ }^{35}$ Department of Epidemiology, University Medical Center of Rotterdam, Rotterdam, GE 3015, The Netherlands. ${ }^{36}$ Department of Ophthalmology, King's College London, London, SE5 9RS, UK. ${ }^{37}$ Department of Ophthlmology, Menzies Institute for Medical Research, University of Tasmania, Tasmania, 7000, Australia. ${ }^{38}$ Ophthalmology, Inselspital, University Hospital Bern, University of Bern, Bern, 3010, Switzerland. ${ }^{39}$ Faculty of Medicine, University of Iceland, Reykjavik, 101, Iceland. ${ }^{40}$ Department of Public Health and Primary Care, University of Cambridge, Cambridge, CB2 1TN, England. ${ }^{41}$ Department of Biochemistry, National University of Singapore, Singapore, 117596, Singapore. ${ }^{42}$ Department of Ophthalmology, Erasmus Medical Center, Rotterdam, GE 3015, The Netherlands. ${ }^{43}$ Clinical and Experimental Sciences, Faculty of Medicine, University of Southampton, Southampton, SO17 1BJ, UK. ${ }^{44}$ Centre for Eye Research Australia, University of Melbourne, Royal Victorian Eye and Ear Hospital, Melbourne, VIC 3002, Australia. ${ }^{45}$ Statistical Genetics, QIMR Berghofer Medical Research Institute Royal Brisbane Hospital, Brisbane, QLD 4006, Australia. ${ }^{46}$ Department of Ophthalmology and Visual Sciences, The Chinese University of Hong Kong, Hong Kong, Hong Kong. ${ }^{47}$ Institute of Human Genetics, Friedrich-Alexander-Universität Erlangen-Nürnberg (FAU), Erlangen, 91054, Germany. ${ }^{48}$ deCODE genetics/Amgen, Inc., Reykjavik, IS-101, Iceland. ${ }^{49}$ MRC Human Genetics Unit, The University of Edinburgh, Edinburgh, EH4 2XU, Scotland.

${ }^{50}$ McPherson Eye Research Institute, University of Wisconsin-Madison, Madison, WI 53705, USA. ${ }^{51}$ Clinic for General and Interventional Cardiology, University Heart Center Hamburg, Hamburg, 20246, Germany 